\title{
Cosmic ray variations in November, 2012
}

\section{Lukovnikova A. A. ${ }^{a}$ *}

a Institute of Solar-Terrestrial Physics, Siberian Branch of the Russian Academy of Sciences,, Lermontov st., 126a, Irkutsk, Russia

E-mail: luk@iszf.irk.ru

Using data of satellite and ground-based cosmic ray (CR) observations at the worldwide network of neutron monitors, Forbush effects (FE) in November 2012 have been studied using the spectrographic global survey (SGS) method. The SGS method allows investigating variations in the rigidity spectrum, anisotropy, and changes in the geomagnetic cutoff rigidity during every hour of observations. Data of ground-based measurements on the worldwide network of neutron monitors (41 stations) averaged over 1-hour intervals, and data from the GOES-15 satellite were used for the analysis. Relative changes in CR intensity with $\mathrm{R}=4 \mathrm{GV}$ in the Geocentric Solar Ecliptic coordinate system for different time points of the period studied are presented.

37th International Cosmic Ray Conference (ICRC 2021)

July 12th-23rd, 2021

Online-Berlin, Germany

\section{*Presenter}

(C) Copyright owned by the author(s) under the terms of the Creative Commons 


\section{Introduction}

November 2012 is characterized by the presence of several active regions in the Sun. On 13.11.2012, an M6.0 flare was detected on the Sun in the active region AR No. 1613 (maximum at 2:04 UT). At this point, the maximum IMF modulus was $\sim 10 \mathrm{nT}$, solar wind (SW) velocity $\sim 460 \mathrm{~km} / \mathrm{s}$, proton density $\sim 20 \mathrm{p} / \mathrm{cm}^{3}$. During the rest of the month, $\mathrm{M}$ and $\mathrm{C}$ class flares occurred. The SW velocity was $\sim 300-700 \mathrm{~km} / \mathrm{s}$, IMF modulus was $\sim 5-20 \mathrm{nT}$.

A total of 11 FEs were observed in November 2012 according to [1-5]. The Dst-index during the FE on 12.11 .12 was $-108 \mathrm{nT}$, and on 14.11 .12 it was $-104 \mathrm{nT}$. For 14.11.12 FE, the source was the 10.11.12 flare in AR 1608, onset at 4:22 UT, maximum at 4:32 UT. The Kpindex reached 6 and the rest of November, the Kp-index was 2-5. In November, the amplitudes of CR modulation were $\sim-8 \%$ at polar, $\sim-4.5 \%$ at mid-latitude, and $\sim-3 \%$ at low-latitude stations.

\subsection{Data and method}

For the calculations, we took data from the neutron monitor stations of the worldwide network of cosmic ray stations equipped with neutron monitors (41 CR stations), averaged over 1-hour intervals and corrected for pressure. The modulation amplitudes for November 2012 were counted from the 01.11.12 background level.

For the analysis, we used the spectrographic global survey (SGS) method [6-8] that was developed in the ISTP SB RAS. The SGS method within the model of CR modulation with regular heliosphere electromagnetic fields, in the joint analysis of ground-based and satellite measurements, allows the study of variations in the rigidity spectrum, anisotropy, and changes in the geomagnetic cutoff rigidities (GCR) during every hour of observation.

\subsection{Results of analysis}

Figure 1 presents data of direct measurements of the following parameters in the interplanetary space, top-to-bottom: IMF modulus, $\mathrm{SW}$ velocities, proton temperature and proton density of the solar wind, time profiles of variations in global CR intensity with rigidity 4 (black line) and $10 \mathrm{GV}$ (red line), changes in the geomagnetic cutoff rigidity $\Delta \mathrm{Rc}$ at the point with threshold rigidity $\mathrm{Rc}=4 \mathrm{GV}$ (red line) together with the Dst-index (black line), amplitudes of the first $A_{1}$ and second $A_{2}$ spherical harmonics of the $C R$ pitch-angle anisotropy for particles with $\mathrm{R}=4 \mathrm{GV}$ in October 2012.

For particles with rigidity $\mathrm{R}=4 \mathrm{GV}$, the maximum modulation amplitude in November 2012 was $\sim-20 \%$, for particles with rigidity $\mathrm{R}=10 \mathrm{GV}$ it was $\sim-6$, as shown in Figure 1 .

For particles with $\mathrm{R}=4 \mathrm{GV}$, the amplitudes of the anisotropy first harmonic $\mathrm{A}_{1}$ observed in November 2012 reached $\sim 25 \%$, and the amplitudes of the second harmonic $\mathrm{A}_{2}$ reached $\sim 6 \%$. A magnetic storm was observed on 14.11.12 (Dst -108 nT).

In Figures 2-3, equilines depict relative changes in the intensity of CRs with $\mathrm{R}=4 \mathrm{GV}$ in the Geocentric Solar Ecliptic coordinate system for different points of time on 14.11.12 (Fig. 2) and 25.11.12 (Fig. 3). Along the X-axis, there are values of the longitude angle $\psi$; along the Yaxis - of the latitude angle. Numerals on the equilines are amplitudes of particle intensity variations as a percentage of the background level. 


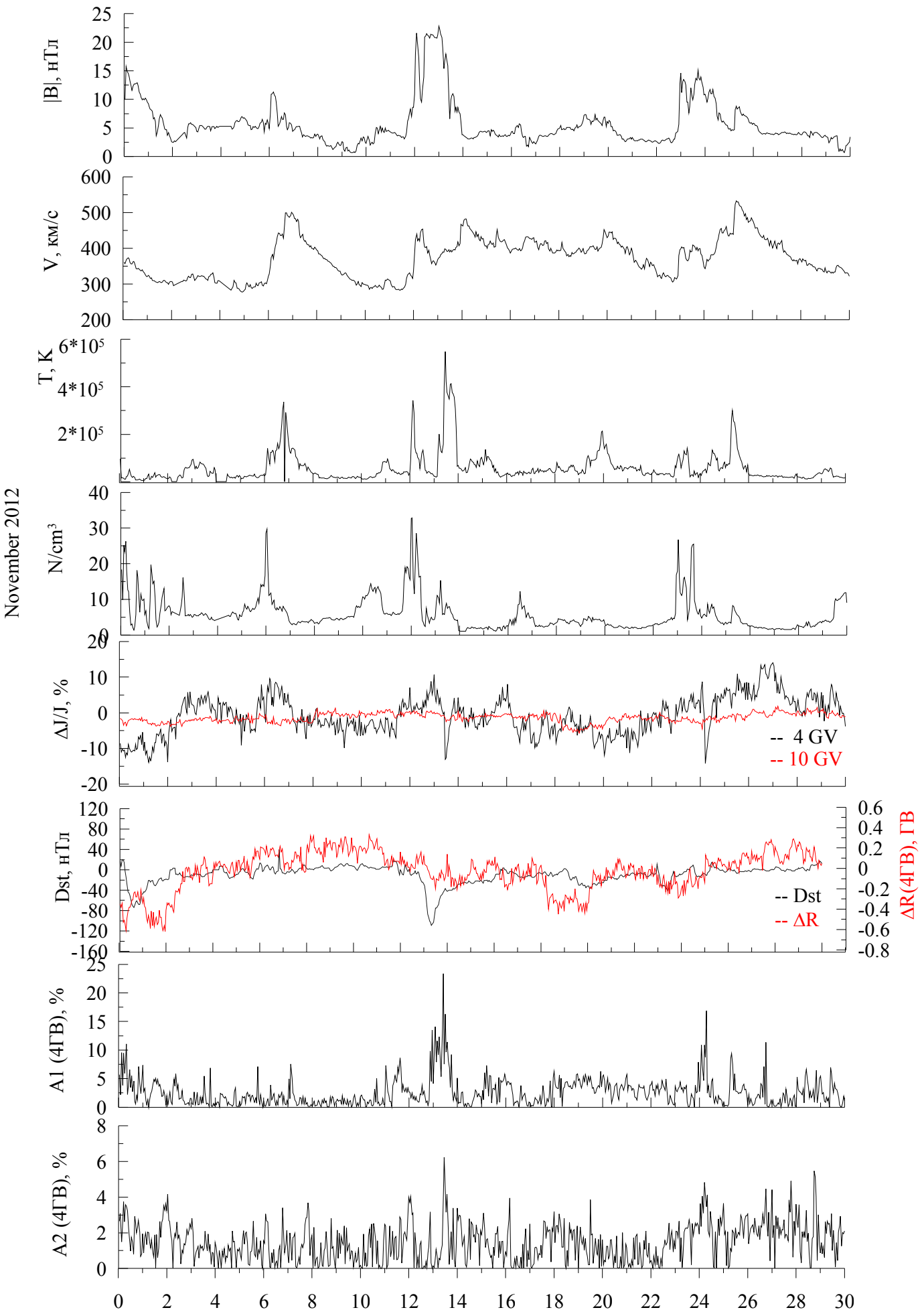

Fig.1 IMF modulus, SW velocity, SW temperature, SW density, time profiles of variations in global CR intensity with rigidity 4 (black line) and $10 \mathrm{GV}$ (red line), changes in the geomagnetic cutoff rigidity $\Delta \mathrm{Rc}$ at the point with threshold rigidity $\mathrm{Rc}=4 \mathrm{GV}$ (red line) together with the Dst-index (black line), amplitudes of the first $A_{1}$ and second $A_{2}$ spherical harmonics of the CR pitch-angle anisotropy for particles with $\mathrm{R}=4 \mathrm{GV}$. 

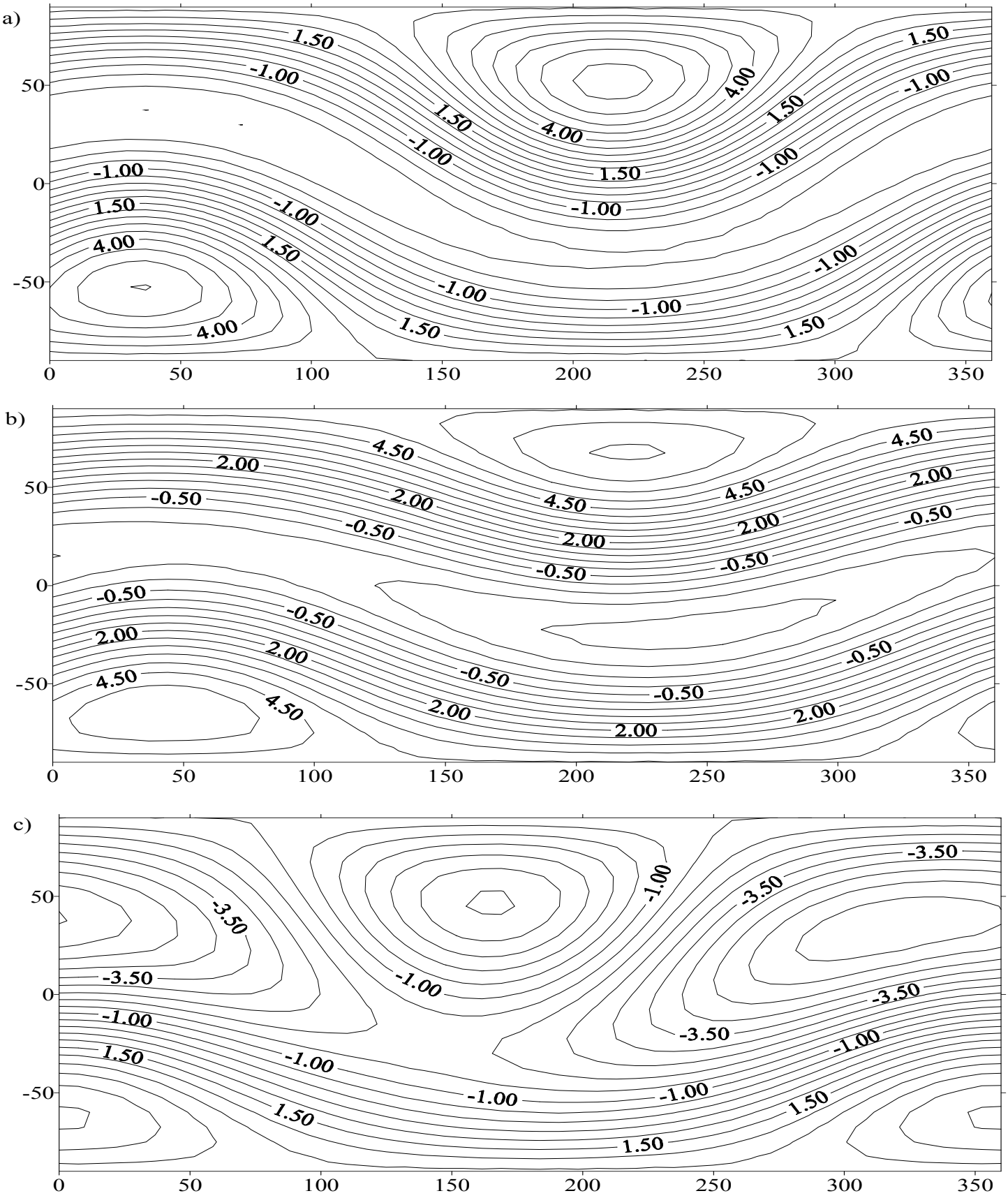

Fig. 2 Relative changes in $\mathrm{CR}$ intensity with $\mathrm{R}=4 \mathrm{GV}$ in the Geocentric Solar Ecliptic coordinate system for different time points on 14 November 2012 ( $\mathrm{a}-$ 09:00 UT, b - 10:00 UT, c - 10:00 UT). Along the X-axis, there are values of the longitude angle $\psi$; along the Y-axis of the latitude angle $\lambda$. 

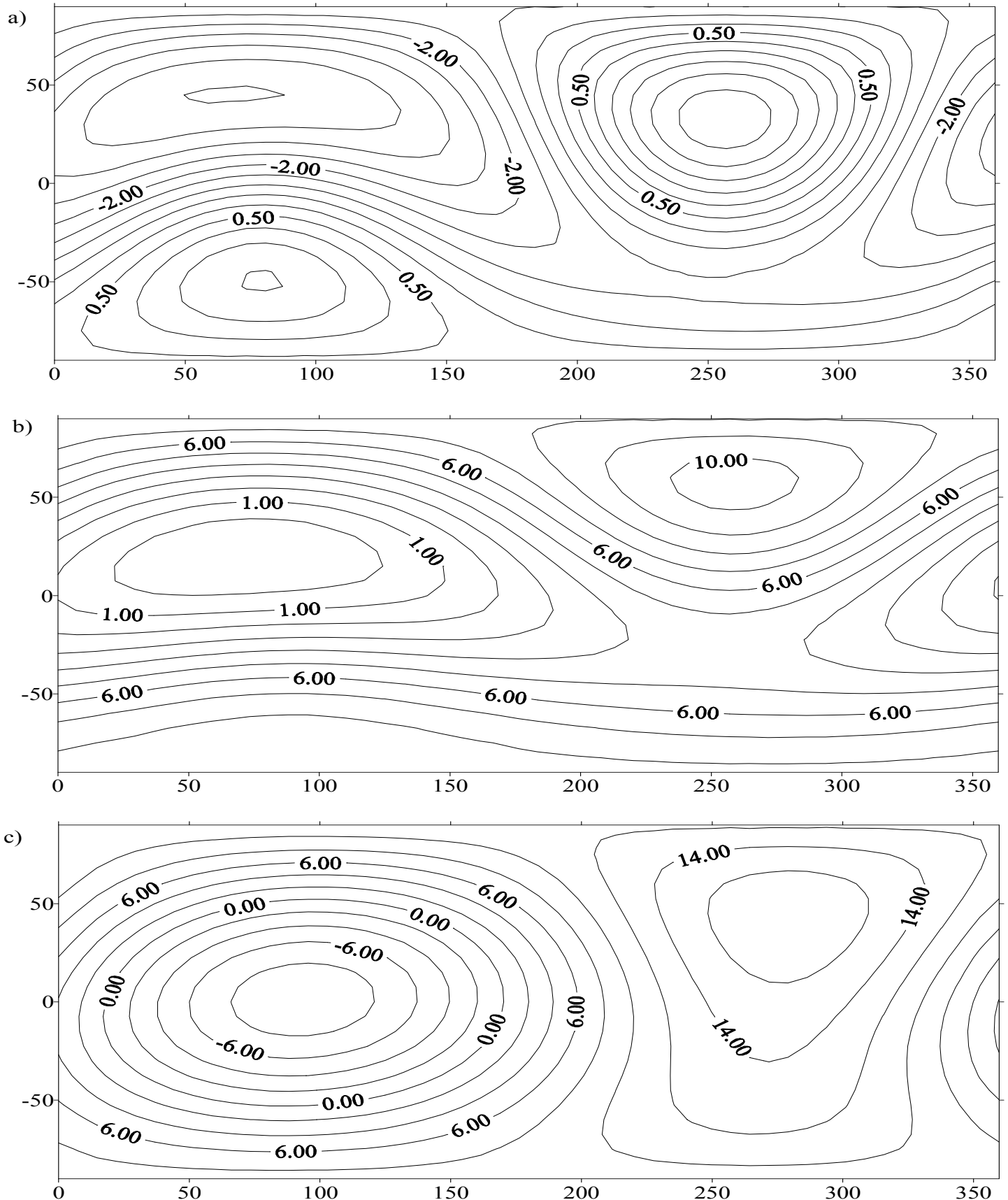

Fig. 3 Relative changes in $\mathrm{CR}$ intensity with $\mathrm{R}=4 \mathrm{GV}$ in the Geocentric Solar Ecliptic coordinate system for different time points on 25 November 2012 ( $\mathrm{a}-$ 03:00 UT, b - 4:00 UT, c 6:00 UT). Along the X-axis, there are values of the longitude angle $\psi$; along the $\mathrm{Y}$-axis - of the latitude angle $\lambda$. 
Figure 2 analysis implies that the 14.11.2012 increase in the CR intensity was observed from directions $\psi \sim 30^{\circ}, 215^{\circ}, \lambda=\sim-50^{\circ}, \sim 45^{\circ}$ (panel a, 09:00 UT), $\psi \sim 40^{\circ}, 210^{\circ}, \lambda=\sim-40^{\circ}$, $\sim 75^{\circ}$ (panel b, 10:00 UT), $\psi \sim 0^{\circ}, 160^{\circ}, \lambda=\sim-30^{\circ}, \sim 50^{\circ}$ (panel c, 11:00 UT). CR intensity from these directions was $\sim 8 \%$ higher than the lowest observed intensity.

As shown in Figure 3, the increase in CR intensity on 25.11 .12 was observed from directions $\psi \sim 80^{\circ}, 260^{\circ}, \lambda=\sim-50^{\circ}, \sim 35^{\circ}$ (panel a, 03:00 UT), $\psi \sim 255^{\circ}, \lambda=\sim 60^{\circ}$ (panel $\mathrm{b}$, 04:00 UT), $\psi \sim 260^{\circ}, \lambda=\sim 45^{\circ}, \sim 0^{\circ}$ (panel c, 06:00 UT). During the CR intensity decrease, the elevated amplitudes of the second harmonic $\mathrm{A}_{2}$ for particles with $\mathrm{R}=4 \mathrm{GV}$ were $\sim 10 \%$. The high-amplitude bidirectional anisotropy in particle angular distribution bespeaks the occurrence of a magnetic trap-like IMF structure related to the Sun [7].

\subsection{Conclusions}

Based on the analysis, it can be concluded that the maximum amplitudes of the anisotropy first harmonic $\mathrm{A}_{1}$ reached $\sim 25 \%$, and the amplitudes of the anisotropy second harmonic $\mathrm{A}_{2}$ reached $\sim 6 \%$ for particles with $\mathrm{R}=4 \mathrm{GV}$. In November 2012, the maximum modulation amplitude for particles with $\mathrm{R}=4 \mathrm{GV}$ was $\sim-5 \%$, with $\mathrm{R}=10 \mathrm{GV} \sim-8 \%$. At the time points when the maximum modulation amplitude for particles with $\mathrm{R}=10 \mathrm{GV}$ is detected, we can observe the magnetic storm with Dst $\sim-120 \mathrm{nT}$, the IMF modulus increase to $30 \mathrm{nT}$, the solar wind velocity increase to $700 \mathrm{~km} / \mathrm{s}$, and Forbush Effects on November 9-15, 2012. The Earth was inside a Sun-related magnetic trap-like IMF loop structure, which deforms the background magnetic field, as evidenced by the appearance of bidirectional anisotropy of great amplitude in the angular distribution of particles.

The work was financially supported by the Ministry of Science and Higher Education of the Russian Federation. The results were obtained using the equipment of Shared Equipment Center “Angara” http://ckp-rf.ru/ckp/3056/.

\section{References}

[1] http://tesis.lebedev.ru/sun_flares.html

[2] http://www.spaceweather.com

[3] http://www.swpc.noaa.gov/Data/goes.html

[4] http://spaceweather.izmiran.ru/rus/fds2012.html

[5] V. N. Ishkov, The Sun in October-November 2012. The Earth and the Universe. 2013. No. 2. P. 33-35.

[6] V.M. Dvornikov, V.E. Sdobnov, A.V. Sergeev, Analysis of cosmic ray pitch - angle anisotropy during the June 1972 Forbush effect by method of spectrografic global survey. Proc. 18th ICRC. 1983. Bangalore. India. V. 3. P. 249-253.

[7] V.M. Dvornikov, V.E. Sdobnov, Variations in the rigidity spectrum and anisotropy of cosmic rays at the period of Forbush effect on 12-15 July. IJGA. 2002. V. 3. No 3. P. 217-226.

[8] I.G. Richardson, V.M. Dvornikov, V.E. Sdobnov et al. Bidirectional particle flows at cosmic ray and lower $(\sim 1 \mathrm{MeV})$ energies and their association with interplanetary coronal mass ejections/ejecta. J. Geophys. Res. 2000. V. 105. No A6. P.12579-12591. 